\title{
DEVELOPMENT AND IN VITRO ASSESSMENT OF BUSPIRONE LOADED IN SITU NANOEMULSION GEL
}

\section{KAMALESH TRIPATHI ${ }^{*}$, NIRANJAN KUMAR MANNA ${ }^{2}$}

1SHEAT College of Pharmacy, Varanasi, Uttar Pradesh, India, ${ }^{2}$ Faculty of Pharmacy, CSM Group of Institutions, Allahabad, Uttar Pradesh, India Email: tripathi1921@gmail.com

Received: 05 Apr 2021, Revised and Accepted: 11 May 2021

\section{ABSTRACT}

Objective: Buspirone, is a medication primarily used for generalized anxiety disorder (GAD), relieve symptoms of anxiety and unipolar depression. This drug exhibit low bioavailability (approximately 5\%) due to extensive first-pass metabolism and non-targeted delivery results in numerous side effects. It is taken by mouth, and it may take up to four weeks to have an effect. The present investigation aimed at the development of buspirone in situ nanoemulsion gel to evaluate its potential for efficacious nose to brain drug delivery.

Methods: Buspirone-loaded nanoemulsions (BNEs) were prepared by aqueous titration (Spontaneous emulsification) method using Oleic acid, Tween 80, and PEG 400 as oil, surfactant and cosurfactant respectively. The NEs (FC1-FC8) were characterized for pharmaceutical characteristics (Appearance, thermodynamic stability, polydispersity index (PDI) value, globule size, pH, Viscosity, Conductivity and Refractive index). In vitro drug release study from nanoemulsions (NEs) was carried out using Keshary-Chien cell (KC cell, 25 ml) in phosphate buffer pH 5.5.

Results: Formulation FC5 with mean globule size of $105.4 \pm 1.10 \mathrm{~nm}$, PDI value $0.230 \pm 0.01$ and drug release $90 \pm 0.39 \%$ in $6 \mathrm{~h}$ (h) was developed as mucoadhesive nanoemulsion gel formulation with $17.5 \% \mathrm{~W} / \mathrm{W}$ of Pluronic F127. The nanoemulsion gel was homogenous, transparent, and possessed a bioadhesive strength of 1605 Dyne/cm2. In vitro cumulative drug release was found to be $90.00 \pm 0.39 \%$ at the end of $6 \mathrm{~h}$.

Conclusion: The gel had no effect on the structural integrity of nasal mucosa. Hence, the study postulates that In situ nanoemulsion gel of buspirone could be used as an intranasal formulation for targeted brain delivery via nasal route.

Keywords: Nanoemulsion, Buspirone, In situ gel, Intranasal, Brain delivery, Phase diagram

(C) 2021 The Authors. Published by Innovare Academic Sciences Pvt Ltd. This is an open access article under the CC BY license (https://creativecommons.org/licenses/by/4.0/) DOI: https://dx.doi.org/10.22159/ijap.2021v13i4.41694. Journal homepage: https://innovareacademics.in/journals/index.php/ijap

\section{INTRODUCTION}

Controlled release formulations for brain targeting via nasal route have received a considerable concern for a variety of reasons. This is because the nasal route offers a direct link between brain and nose resulting in reduced drug concentration at the non-target site (which reduces the drug toxicity) and higher drug concentration in the brain[1, 2] thus improving therapeutic efficiency which overcomes the limitation of conventional drug delivery systems.

Buspirone[8-(4-(4-(2-Pyrimidinyl)-1-piperizinyl)butyl)-8-azaspiro $(4,5)$ decane-7,9-dione] is an approved anxiolytic agent belonging to the chemical class of azaspirodecanedione and is available as a tablet (Buspar $\AA$, Anxiron $₫$, Anxut $\AA$, Bespar $®$ ) used for generalized anxiety disorder (GAD), relieve symptoms of anxiety and for unipolar depression[3]. This dosage form exhibit low bioavailability (approximately 5\%) due to extensive first-pass metabolism and nontargeted delivery results in numerous side effects, such as dizziness, drowsiness, headache, nausea, nervousness, lightheadedness, restlessness, blurred vision, tiredness, and trouble sleeping may occur [4].

Nanotechnology involves the fabrication of nanosystems that deliver drugs in a sustained and controlled manner. These nanodevices include colloidal carriers like lipid (solid lipid nanoparticles (SLN), nanoemulsions, liposomes, and nanostructured lipid carriers (NLC) etc.) and polymeric nanoparticles (chitosan nanoparticles, PLGA nanoparticles [5]. Nanoemulsions are thermodynamically stable transparent (translucent) dispersions of oil and water stabilized by an interfacial film of surfactant and cosurfactant molecules having a droplet size of less than $100 \mathrm{~nm}$. Nanoemulsions have the possibility to improve the absorption, penetration, efficiency and safety of the therapeutic agent to a great extent [5-7]. Studies have shown that nanoemulsion formulations possess improved transdermal and dermal delivery properties in vitro and in vivo [6].

In situ nanoemulsion gel formulation increases the nasal permeation of therapeutic moiety to the brain, improves delivery at a constant and slow release rate, protect therapeutics from degradation along the pathway, increases mucoadhesion and facilitate overall nasal transport [9-11].

The aim of this study was to develop a BNEs based in situ gel and evaluate its potential for brain targeting via nasal route. The nanoemulsion gel was prepared by dispersing the BNEs in thermoreversible polymer Pluronic F127 gel base, and its efficacy was evaluated by performing an ex vivo diffusion study across goat nasal mucosa in phosphate buffer saline (PBS) pH 5.5.

\section{MATERIALS AND METHODS}

\section{Materials}

Buspirone was procured from Yarrow Chem Products, India. Pluronic F127 (PF127) was acquired from BASF, (Germany). Tween 80, Triton X 100, Tween 20, Oleic acid, Triacetin, Potassium dihydrogen phosphate, Isopropyl myristate (IPM), Ethanol, Propanol, Polyethylene glycol 400 (PEG 400) and Propylene glycol were procured from SD Fine-Chem Limited (Ahmedabad, India). All other reagents and solvents used were of analytical grade.

\section{Methods}

Formulation development of nanoemulsion

NEs were formulated by aqueous phase titration method (Spontaneous emulsification) which involved a process of addition of water from burette to the solution i.e. the mixture of surfactant, co-surfactant and oil present in beaker till a transparent solution is obtained. The significant yardstick for the selection of ingredients for NEs is the solubility of the poorly soluble drug in surfactant, oil and cosurfactant $[8,9,13]$.

\section{Selection of oil, surfactant and co-surfactant}

Oil, surfactant and co-surfactant were selected by performing a solubility study $[9,11]$. The drug was dissolved in oil phase [Ethyl Oleate, IPM, Oleic Acid, Olive Oil, Sesame Oil, Triacetin, 
Triacetin+IPM (1:1), Triacetin+Ethyl Oleate (1:1)], surfactant (Tween 80, Triton X100, Tween 20, Labrafil) and co-surfactant (PEG 400, Ethanol, Propanol and Propylene glycol) till saturation. Oil, surfactant and co-surfactant were selected which gave maximum solubility of the drug (table 2).

\section{Pseudo ternary phase diagrams}

On the basis of the solubility studies, a combination of Oleic acid, Tween-80 and PEG-400 were selected as oil, surfactant and cosurfactant, respectively. Distilled water was used as an aqueous phase. Surfactant and co-surfactant (Smix) were mixed at different mass ratios (1:0, 1:1, 2:1 and 1:2). These ratios were chosen in increasing concentration of surfactant with respect to co-surfactant and increasing concentration of cosurfactant with respect to surfactant for a detailed study of the phase diagrams. For each phase diagram, oil and Smix at a specific ratio were mixed thoroughly at different mass ratios from 9:1, 1:0.25, 1:0.43, 1:1, 1:2, 1:3, 1:4, 1:5, $1: 9(\mathrm{w} / \mathrm{w})$ in different glass vials. Pseudo ternary phase diagrams of oil, Smix and aqueous phase were developed using the aqueous titration method. Slow titration with an aqueous phase was performed for each mass ratio of oil and Smix and visual observations were made for transparent and easily flowable $\mathrm{o} / \mathrm{w}$ nanoemulsions. The pseudo ternary phase diagrams were developed by using CHEMIX School Ver. 3.50 software (MN, USA) (fig. 2)[15]. The composition of nanoemulsion in the phase diagrams was depicted by the following equation.

$$
\begin{gathered}
\% \text { Oil (Oleic acid })+\% \text { Smix }(\text { Tween } 80+\text { PEG } 400)+\% \text { water }= \\
100 \text { Eq. } 1
\end{gathered}
$$

\section{Preparation of nanoemulsion}

NEs containing buspirone were formulated by the aqueous phase titration method (Spontaneous emulsification) [14]. The specified quantity of the drug was dissolved in oil phase under stirring pursued by addition of $\operatorname{Smix}(1: 1)$ in a fixed proportion by vortexing (by vortex mixer) pursued by water addition from a burette to the solution (Smix+Oil+Buspirone) present in beaker till a transparent solution was obtained (table 1).

\section{Dispersion stability studies}

To overcome the problem of metastable formulation, thermodynamic stability tests were performed. Formulations were centrifuged at $3500 \mathrm{rpm}$ for $30 \mathrm{~min}$. and then subjected to six cycles of heating cooling analysis between the refrigerator temperature (4 ${ }^{\circ} \mathrm{C}$ ) and $45^{\circ} \mathrm{C}$ with storage at each temperature for not less than $48 \mathrm{~h}$ were done [12].

\section{Characterization of nanoemulsions}

\section{Qualitative study}

In the dilution test, $1 \mathrm{ml}$ of nanoemulsion was diluted to $100 \mathrm{ml}$ with purified water and checked for clarity of formulation. In another test, type of nanoemulsion was identified by sprinkling a watersoluble dye (Methyl orange) over nanoemulsion and observed visually. All the formulations were subjected to a centrifugation cycle of $15 \mathrm{~min}$ and assessed for whether the nanoemulsion was monophasic or biphasic [11].

\section{Morphology}

Morphology and structure of the NEs were studied using transmission electron microscopy TOPCON 002B operating at 200 $\mathrm{KV}$ (Topcon, USA) and capable of point-to-point resolution. A Combination of bright field imaging at increasing magnification and of diffraction modes was used to reveal the form and size of nanoemulsion droplets. In order to perform the transmission electron microscopy (TEM) observations, a drop of the nanoemulsion was directly deposited on the holey film grid and observed after drying [13].

\section{Droplet size analysis}

The droplet size distribution of the nanoemulsion was determined by photon correlation spectroscopy, using a Zetasizer $1000 \mathrm{HS}$ (Malvern Instruments, UK). Light scattering was monitored at $25^{\circ} \mathrm{C}$ at a $90^{\circ}$ angle. Droplet size distribution studies were performed at a fixed refractive index of the respective formulation [14].

\section{$\mathrm{pH}$, viscosity, conductivity and refractive index}

The $\mathrm{pH}$ of the nanoemulsions was measured by using a $\mathrm{pH}$ meter (PM608 model-Analion, Brazil) at $25 \pm 2{ }^{\circ} \mathrm{C}$. The viscosity of the formulations was determined as such without dilution using a Brookfield viscometer (RV model with spindle No. 3) at room temperature. The electrical conductivity was evaluated at $25 \pm 2{ }^{\circ} \mathrm{C}$ by a conductivity meter (mCA-150 model, Tecnopon, Brazil). Refractive index of the formulations was determined using an Abbes type refractometer $[15,18]$.

\section{Zeta potential}

Zeta potential is the potential difference between the dispersion medium and the stationary layer of fluid attached to the dispersed particle. Its value can be related to the stability of colloidal dispersions. Zeta potential was determined using a Zetasizer 1000 HS (Malvern Instruments, UK) [16].

\section{In vitro permeation study}

In vitro permeation study across goat nasal mucosa was performed on a modified KC diffusion cell of $25 \mathrm{ml}$ of receiver chamber capacity. The mucosa was mounted between the donor and receiver compartments of the Keshary-Chien diffusion cell. $1 \mathrm{ml}$ nanoemulsion formulation containing buspirone was placed into the donor compartment and phosphate buffer $\mathrm{pH} 5.5$ was added to the acceptor compartment. Samples were withdrawn at regular intervals $(15,30,60,120$ and $180 \mathrm{~min})$, filtered through $0.45 \mu \mathrm{m}$ membrane filter and analyzed for drug content by UV-Vis Spectrophotometer at $238 \mathrm{~nm}$ [17].

\section{Selection of optimized nanoemulsion formulation}

The nanoemulsion formulation was optimized on the basis of stability, lowest PDI value, optimum particle size and percentage of cumulative drug permeated.

\section{Preparation of buspirone loaded in situ nanoemulsion gel}

Gels containing various concentrations of PF 127 were prepared by cold method and optimized on the basis of gelation time and gelation temperature. Specified quantity of PF 127 (15.5\%, 16.0\%, 16.5\%, $17.0 \%$ and $17.5 \% \mathrm{w} / \mathrm{w}$ ) was added into deionized water at $4{ }^{\circ} \mathrm{C}$ under stirring. The formulations were refrigerated up to $12 \mathrm{~h}$ till the complete dissolution of PF 127 was accomplished which was confirmed by a transparent solution at $4{ }^{\circ} \mathrm{C}$. Nanoemulsion gel formulation (FC5) was formulated in $17.5 \%(\mathrm{w} / \mathrm{w})$ of Pluronic PF 127 in deionised water pursued by the addition of Benzalkonium chloride $(0.001 \%)$ by using the cold method [23-27].

\section{Clarity, $\mathrm{pH}$, gelation temperature and gelation time}

The naoemulsion gel formulation (FCG5) was then evaluated for clarity, $\mathrm{pH}$, gelation temperature and gelation time. The clarity of the optimized gel formulations was determined before and after gelation by visual examination of the formulations under light, alternatively against the white and black background. The $\mathrm{pH}$ of the developed gel was determined using a digital probe $\mathrm{pH}$ meter. The sol gel transition temperature was evaluated by transferring $2 \mathrm{ml}$ of formulation to a test tube. After sealing with a parafilm, the test tube was kept in a circulation waterbath at $37^{\circ} \mathrm{C}$. Following each temperature setting, equilibration was allowed for $10 \mathrm{~min}$. Finally, the test tube was placed horizontally to observe the state of the sample and to examine the gelation [11, 28, 29].

\section{Mucoadhesion studies}

The mucoadhesive strength of preparation was resolved by calculating the mandatory force to separate the preparation from nasal mucosal tissue. A segment of goat nasal mucosa was fitted in both the slides with the help of thread. After that $50 \mathrm{mg}$ of gel was put on the first slide and locate this slide below the heightadjustable pan and put the other slide with the mucosal portion beneath the same pan but in reverse position. The two slides have adhered in such a way that the gel present in between touches each 
other. This should be done at least for 2 min to ascertain the confidential contact between them. The weights were held on to rising continuously in the second pan and did until the slides get separated from one another. The mucoadhesive forces revealed as the attachment stress in (dyne $\left./ \mathrm{cm}^{2}\right)$ was decisively from the least possible weight that isolated the mucosal tissue from the superficial part of each formulation [19].

$$
\text { Mucoadhesive Potential }=\frac{m g}{A} \text { Eq. } 2
$$

Where, $\mathrm{m}=$ weight required for detachment (in gram), $\mathrm{g}=$ Acceleration due to gravity, A = Area of mucosa exposed.

\section{Ex vivo diffusion study}

The freshed cut-out goat nasal mucosa not having septum part was taken from slaughterhouse in PBS pH 5.5 and kept for 15 min to bring in to equilibrium. The superior scrolled nasal conche or turbinate was diagnosed and segregated from the nasal membrane. The eliminated superior nasal membrane was organized on KC cell. The tissue was maintained at the unfluctuating level by using phosphate buffer and let on to agitate for $15 \mathrm{~min}$ by using a magnetic stirrer. After $15 \mathrm{~min}$ the solution was taken away from both the compartment. From these on, cramed fresh phosphate buffer into the accepter compartment.

One ml of nanoemulsion gel was put in the donor compartment of KC cell in addition to $1.7 \mathrm{ml}$ of phosphate buffer. Sample from receptor compartment was taken at the predetermined time period and examined, was replaced by the same volume of diffusion media. The study was accomplished in six $\mathrm{h}$, at the same time the amount of drug $(\mu \mathrm{g} / \mathrm{ml})$ in the acceptor chamber, against the goat nasal membrane, was measured at each sampling point [20].

\section{Test for nasal cilio toxicity of nanoemulsion gel}

There were selected three goats nasal mucosa namely A1, A2 and A3 with constant width and were organized on KC cell. A1 was prepared with $0.5 \mathrm{ml}$ of Phosphate buffer (negative control, $\mathrm{pH}$ 6.4), A2 with $0.5 \mathrm{ml}$ of Isopropyl alcohol (Positive control) and A3 was prepared with nanoemulsion gel by exposing them for one $h$. Behind in $1 \mathrm{~h}$ time, the mucosa was washed out with buffer and exposed to histological examination to measure out the toxicities of preparation on photographed microscope [14].

\section{Mechanism of drug release}

To study release kinetics, data obtained from In vitro percentage cumulative drug release studies were fitted in different kinetic model i.e. cumulative percentage of drug released versus time (Zero order), log of cumulative percentage of drug remaining versus time (first-order) and cumulative percentage of drug released versus square root of time (Higuchi's model) and log percentage drug released vs log time (Korsmeyer Peppas) [21,30].

\section{RESULTS AND DISCUSSION}

\section{Solubility studies}

Data obtained from the solubility study of buspirone in various components are depicted in table 2 . All the excipients chosen had shown a better solubility for buspirone (fig. 1). The solubility of buspirone was highest in order (Oleic Acid $>$ Triacetin $>$ Triacetin + IPM (1:1) $>$ Ethyl Oleate $>$ Sesame oil, Triacetin+Ethyl Oleate $>$ Olive Oil>IPM), (Tween 80>Labrafil $>$ Triton $X$ 100>Tween 20) and (Ethanol $>$ Propanol $>$ PG $>$ PEG 400) in oil phase, surfactant and cosurfactant respectively. The selection of the right excipient towards the effective formulation of nanoemulsion is mostly dependent on the solubility of the drug in excipients. Oleic acid was preferred as an oil phase for the formation of nanoemulsion due to its lightweight and higher solubility by which it required less amount of surfactant which reduces irritation. In present work, PEG 400 and Tween 80 were preferred as cosurfactant and surfactant respectively because the formation of an ideal $0 / \mathrm{W}$ nanoemulsion should require criteria such as a combination of surfactant and cosurfactant for the development of fluid interfacial film, desired hydrophilic-lipophilic balance (HLB) value shall be above than ten, the right combination of high and low HLB value surfactant and the existence of cosurfactants to decrease the bending stress of interface which provides sufficient flexibility to interfacial film to take different curvatures needed to make NEs [22].

Table 1: Composition of nanoemulsion

\begin{tabular}{|c|c|c|c|c|c|}
\hline Formulation code & Oil: Smix & Oil $(\% w / w)$ & Surf. (\%w/w) & Co-surf. (\%w/w) & Water(\% w/w) \\
\hline FC 1 & $1: 0.25$ & 5.80 & 0.725 & 0.725 & 92.75 \\
\hline FC 2 & $1: 0.43$ & 5.88 & 1.265 & 1.265 & 91.59 \\
\hline FC 3 & $1: 1$ & 6.69 & 3.345 & 3.345 & 86.62 \\
\hline FC 4 & $1: 2$ & 6.14 & 6.14 & 6.14 & 81.58 \\
\hline FC 5 & $1: 3$ & 6.91 & 10.365 & 10.365 & 72.36 \\
\hline FC 6 & $1: 4$ & 7.80 & 15.60 & 15.60 & 82.95 \\
\hline FC 7 & $1: 5$ & 16.10 & 40.235 & 40.235 & 3.43 \\
\hline FC 8 & 1:9 & 9.79 & 44.21 & 44.21 & 1.79 \\
\hline
\end{tabular}

Table 2: Solubility of drug in surfactant*, co surfactant** and oil ${ }^{* * *}$ (part of solvent/g)

\begin{tabular}{|c|c|c|c|}
\hline *Triton X 100 & $3.38 \pm 0.006$ & $* * *$ Ethyl Oleate & $62.41 \pm 0.09$ \\
\hline *Tween 80 & $2.06 \pm 0.06$ & $* * *$ IPM & $341.09 \pm 1.05$ \\
\hline *Tween 20 & $19.18 \pm 0.16$ & $* * *$ Oleic acid & $5.24 \pm 0.02$ \\
\hline$*$ Labrafil & $2.95 \pm 0.006$ & $* * *$ Olive oil & $202.95 \pm 1.25$ \\
\hline **PEG 400 & $68.86 \pm 0.45$ & $* * *$ Triacetin & $33.3 \pm 0.11$ \\
\hline **Ethanol & $5.3 \pm 0.06$ & *** Sesame oil & $64.17 \pm 0.13$ \\
\hline **Propanol & $6.18 \pm 0.11$ & $* * *$ Tri+IPM $(1: 1)$ & $54.95 \pm 0.65$ \\
\hline$* * \mathrm{PG}$ & $6.97 \pm 0.49$ & ***Tri+Ethyl Oleate $(1: 1)$ & $67.43 \pm 0.24$ \\
\hline
\end{tabular}

Data represents mean \pm SD $(n=3)$

The pseudo ternary phase diagram (selection of surfactantcosurfactant ratio)

Phase diagrams (fig. 2) were designed by varying oil to Smix ratio from 9:1 to 1:9 at Smix ratio 1:0, 1:1, 1:2, 2:1. Nanoemulsion region was displayed by shaded portion in the phase diagram, whereas emulsion region was shown by non-shaded section. Thus ternary phase system of Oleic acid: Tween 80: PEG 400 at Smix (1:1) which exhibiting maximum area was optimized for nanoemulsion (fig. 2b). The ternary diagram shows a significant role in the selection of the formulations to avoid metastable formulations having minimum wetting agent concentration, within the least potential time. The Aqueous phase titration method was preferred for formulation development because it contributes the development of formulation with high thermal stability and drug loading capacity. 


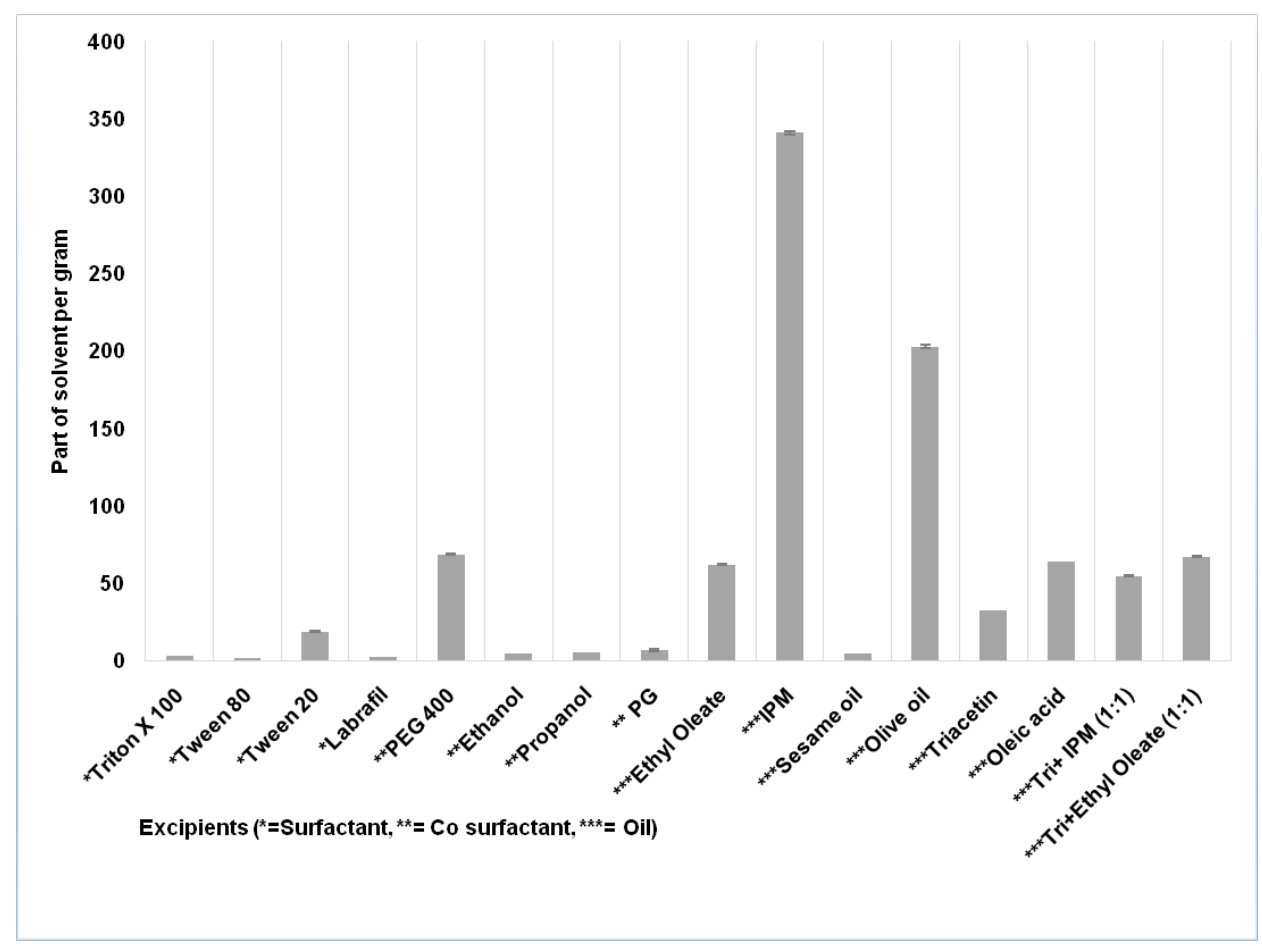

Fig. 1: Solubility of Buspirone in surfactant, cosurfactant and oil, Data represents mean $\pm S D(n=3)$

phase digram at Smix 1:0

phase digram at Smix 1:1

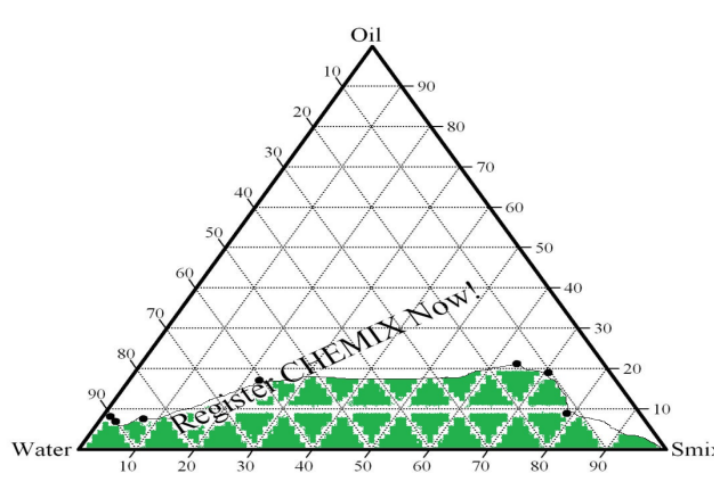

A

phase digram at Smix 1:2

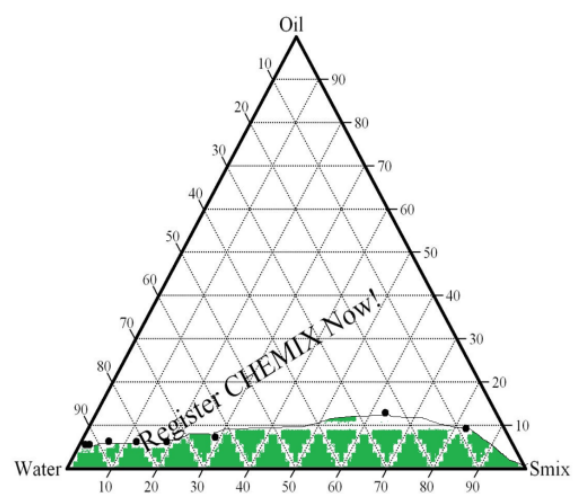

C

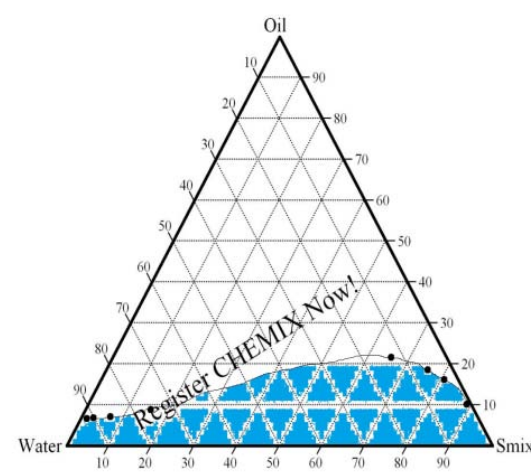

B

phase digram at Smix 2:1

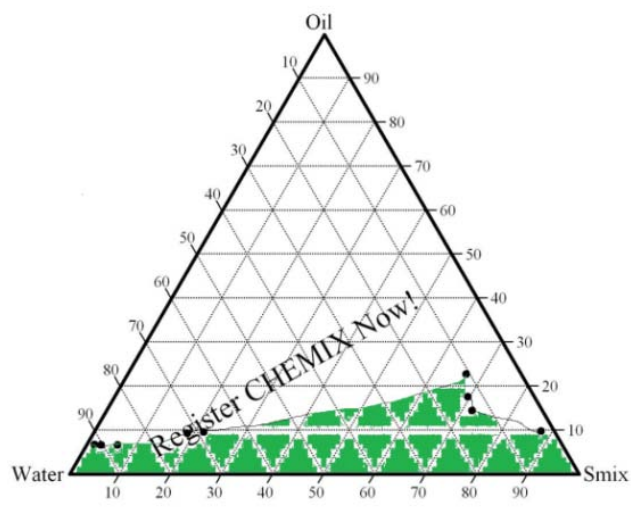

D

Fig. 2: Phase diagram at Smix ratio (A) 1:0, (B) 1:1, (C) 1:2 and (D) 2:1 


\section{Characterization of nanoemulsion}

BNEs were characterized by qualitative and quantitative tests [17]. Transformation in globule size was not shown by nanoemulsion upon dilution and the formulation retained its clarity assuring to be an $\mathrm{O} / \mathrm{W}$ nanoemulsion. The characteristic of nanoemulsion is interpreted by the nature of internal and external phases. In genral, external phase or continuous phase is miscible with the liquid that forms external phase and study was also supported by rapid incorporation of methyl orange (a water-soluble dye) without clumping with continuous phase hence proving that nanoemulsion is of $\mathrm{O} / \mathrm{W}$ type.

Upon centrifugation, neither creaming nor phase separation was observed, indicating the stability of nanoemulsion. All batches passed the stability cycle test. The results of quantitative tests for nanoemulsion are depicted in table 3 and 4 . The $\mathrm{pH}$ of prepared batches of nanoemulsion ranged between 4.25 and 7.00, approximating the normal $\mathrm{pH}$ range of nasal fluids, a percentage transmittance of more than $99 \%$ for nanoemulsion indicated clear dispersion. $\mathrm{pH}$ range shown by formulations indicates that these formulations may relieve in diminishing the irritation potential upon instillation. Conductivity values rely on the higher conductivity of the aqueous phase compared with the oil phase and indicate a higher conductivity value of oil-water nanoemulsion where water is the external phase and indicates nanoemulsion is of o/w type.

The formulation was optimized on the basis of PDI, charge and Size. Optimized formulation FC 5 was selected which has $105.4 \pm 1.10 \mathrm{~nm}$ in size, PDI value $0.230 \pm 0.01$ and charge of $10.4 \mathrm{mv}$ (table 3 ).

Due to the non-ionic nature of excipients, low zeta potential could be associated with the nature of the drug molecule. Physical stability of nanoemulsion system is conferred by zeta potential value of 0 to 10 $\mathrm{mv}$ on the globules of nanoemulsion batches and destabilization of the formulation may be observed above- $10 \mathrm{mv}$. The narrow globule size range and PDI proved that the nanoemulsion approached a stable monodispersed system and could release the drug adequately owing to the larger surface area [23].

Optimized formulation (FC5) was a transparent, clear, monophasic system which confirmed as o/w type of nanoemulsion by dye test. The optimized batch was characterized by $\mathrm{pH} 4.98 \pm 0.03$, viscosity $3.30 \pm 0.01 \mathrm{cps}$, conductance $11.94 \pm 0.03$ mho, refractive index $1.437 \pm 0.002$ (TABLE 3), transmittance $99.5 \%$ and In vitro drug release of $88.05 \pm 0.22 \%$ (table 4 , fig. 3)[24].

Table 3: Quantitative characterization of nanoemulsion

\begin{tabular}{|c|c|c|c|c|c|c|c|}
\hline $\begin{array}{l}\text { Formulation } \\
\text { code }\end{array}$ & $\mathbf{p H}^{*}$ & $\begin{array}{l}\text { Viscosity* } \\
\text { (poise) }\end{array}$ & $\begin{array}{l}\text { Conductivity* } \\
\text { (mho) }\end{array}$ & $\begin{array}{l}\text { Refractive } \\
{ }^{*} \text { index }\end{array}$ & Size $^{*}(\mathrm{~nm})$ & PDI* & $\begin{array}{l}\text { Charge } \\
\text { (mv) }\end{array}$ \\
\hline FC 1 & $6.93 \pm 0.03$ & $1.10 \pm 0.002$ & $8.5 \pm 0.06$ & $1.337 \pm 0.002$ & $273.2 \pm 1.30$ & $0.785 \pm 0.01$ & -3.88 \\
\hline FC 2 & $6.99 \pm 0.16$ & $1.20 \pm 0.002$ & $7.93 \pm 0.03$ & $1.343 \pm 0.003$ & $213.6 \pm 3.06$ & $0.621 \pm 0.01$ & -4.88 \\
\hline FC 3 & $6.90 \pm 0.10$ & $1.45 \pm 0.03$ & $9.93 \pm 0.03$ & $1.352 \pm 0.001$ & $275.9 \pm 2.31$ & $0.335 \pm 0.01$ & -2.12 \\
\hline FC 4 & $5.75 \pm 0.05$ & $1.72 \pm 0.03$ & $11.22 \pm 0.02$ & $1.352 \pm 0.002$ & $389.6 \pm 2.21$ & $0.211 \pm 0.01$ & -0.711 \\
\hline FC 5 & $4.98 \pm 0.03$ & $3.30 \pm 0.01$ & $11.94 \pm 0.03$ & $1.437 \pm 0.002$ & $105.4 \pm 1.10$ & $0.230 \pm 0.01$ & 10.4 \\
\hline FC 6 & $4.25 \pm 0.05$ & $8.16 \pm 0.02$ & $12.39 \pm 0.14$ & $1.449 \pm 0.001$ & $193.9 \pm 2.11$ & $0.303 \pm 0.01$ & 9.16 \\
\hline FC 7 & $5.31 \pm 0.02$ & $15.58 \pm 0.03$ & $5.62 \pm 0.03$ & $1.459 \pm 0.001$ & $689.8 \pm 4.83$ & $0.523 \pm 0.01$ & 5.12 \\
\hline FC 8 & $5.39 \pm 0.04$ & $19.65 \pm 0.05$ & $4.94 \pm 0.02$ & $1.469 \pm 0.001$ & $273.0 \pm 1.90$ & $0.289 \pm 0.01$ & 2.62 \\
\hline
\end{tabular}

Data represents mean $\pm S D(n=3)$

Table 4: In vitro \% release of nanoemulsion

\begin{tabular}{|c|c|c|c|c|c|c|c|c|}
\hline Formulation & FC1 & FC2 & FC3 & FC4 & FC5 & FC6 & FC7 & FC8 \\
\hline \multicolumn{9}{|c|}{ Time (min) $\downarrow$} \\
\hline 0 & 0 & 0 & 0 & 0 & 0 & 0 & 0 & 0 \\
\hline 15 & $11.83 \pm 0.29$ & $13.27 \pm 0.38$ & $11.06 \pm 0.51$ & $12.17 \pm 0.29$ & $14.21 \pm 0.22$ & $11.08 \pm 0.14$ & $13.33 \pm 0.48$ & $19.11 \pm 0.38$ \\
\hline 30 & $25.16 \pm 0.29$ & $27.74 \pm 0.39$ & $19.81 \pm 0.71$ & $25.17 \pm 0.29$ & $34.71 \pm 0.66$ & $19.92 \pm 0.29$ & $28.41 \pm 0.36$ & $36.89 \pm 0.77$ \\
\hline 60 & $43.42 \pm 0.29$ & $48.9 \pm 0.38$ & $34.84 \pm 0.23$ & $40.50 \pm 0.00$ & $41.89 \pm 0.38$ & $29.58 \pm 0.14$ & $41.51 \pm 0.28$ & $50.45 \pm 0.39$ \\
\hline 120 & $75.83 \pm 1.44$ & $70.94 \pm 0.83$ & $64.9 \pm 0.39$ & $68.00 \pm 0.25$ & $53.33 \pm 0.95$ & $48.67 \pm 0.29$ & $63.17 \pm 0.27$ & $70.45 \pm 0.39$ \\
\hline 180 & $90.17 \pm 0.72$ & $87.28 \pm 0.38$ & $87.12 \pm 0.30$ & $82.92 \pm 0.29$ & $88.05 \pm 0.22$ & $82.08 \pm 0.29$ & $85.91 \pm 0.74$ & $81.78 \pm 0.39$ \\
\hline
\end{tabular}

Data represents mean \pm SD $(n=3)$

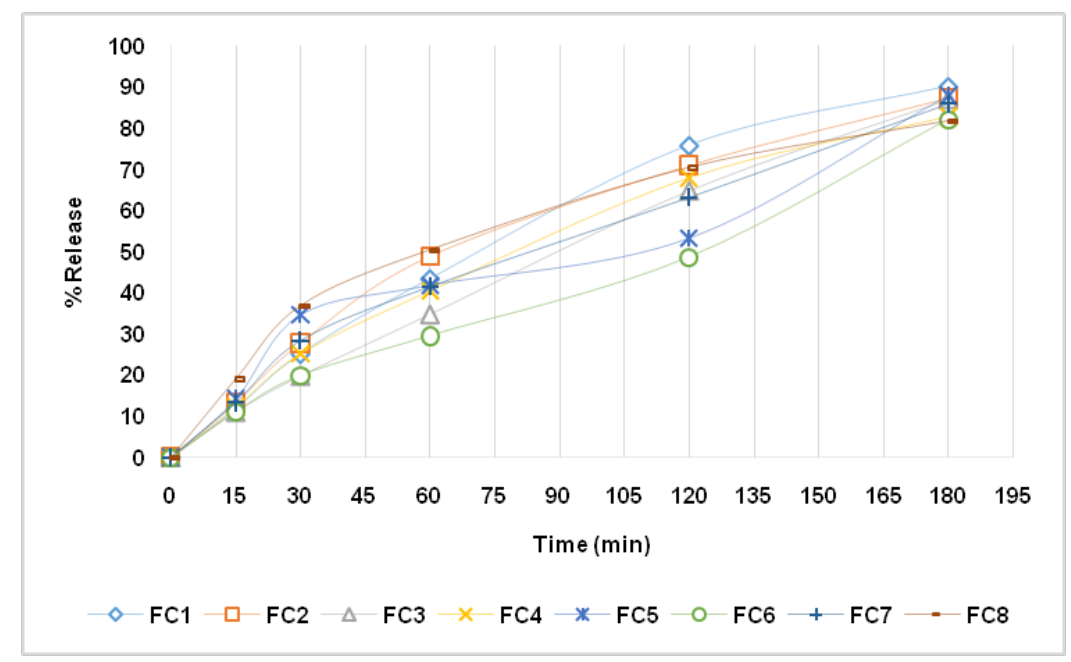

Fig. 3: In vitro release of nanoemulsion, data represents mean $\pm S D(n=3)$ 


\section{Nanoemulsion gel and evaluation}

Preliminary studies indicated that $15-17 \%$ W/W PF-127 formed gel below $38{ }^{\circ} \mathrm{C}$. In this study, $17.5 \%$ concentration of PF 127 was optimized which formed gel at $34{ }^{\circ} \mathrm{C}$. It was found that the formed gel was homogenous, transparent having $\mathrm{pH}$ 4.6. Reduction in gelation temperature was observed with the increase in the concentration of PF-127 leads to the formation of micelle pursued by micellar aggregation and above micellar concentration solution converts into gel phase. At cold temperature, the hydrophobic segment of PF 127 keeps apart due to hydrogen bonding and an increase in temperature leads to disruption of the hydrogen bonds and gel is formed due to hydrophobic interactions. The gelling capacity of PF-127 is directly proportional to the hydrophobic portion, so an increase in the hydrophobic portion leads to the formation of gel [25]. The solution formed gel at $34{ }^{\circ} \mathrm{C}$ in 12 seconds and possesses bio adhesive strength of 1605 Dyne $/ \mathrm{cm}^{2}$. The drug content in the gel was $94.94 \%$. In vitro cumulative drug release was found to be $90.00 \pm 0.39 \%$ at the end of six h (fig. 4). In vitro release and characteristic parameters of optimized in situ nanoemulsion gel formulation confirm its suitability for brain delivery via nasal administration

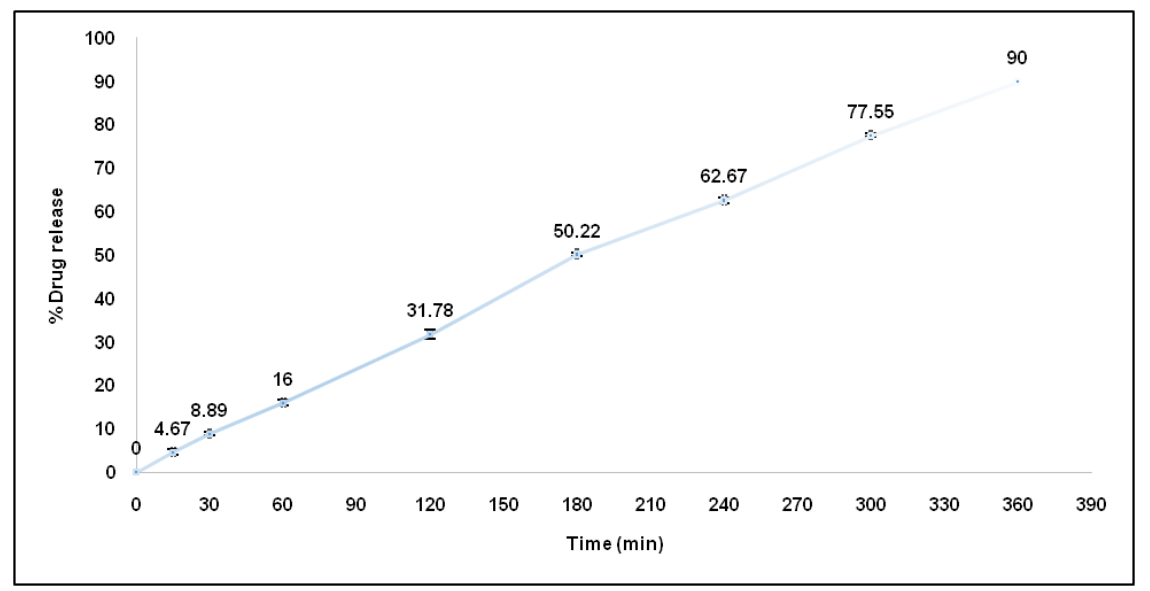

Fig. 4: \% Cumulative drug release of optimized nanoemulsion gel formulation, Data represents mean \pm SD $(n=3)$

\section{Nasal ciliotoxicity studies}

The effect of optimized nanoemulsion gel on the structural integrity of nasal mucosa was assessed by the histological section. Damage to the nasal mucosa with loss of cilia was observed with positive control whereas the nasal mucosa treated with negative control (PBS pH 6.4) and nanoemulsion gel could not show any sign of damage thus confirming the safety of the ingredients used in the nanoemulsion (fig. 5AandB). The effect of optimized nanoemulsion gel on the structural integrity of nasal mucosa was evaluated using histological sections and results confirmed the safety of in situ nanoemulsion gel formulation on nasal membrane structure and integrity.

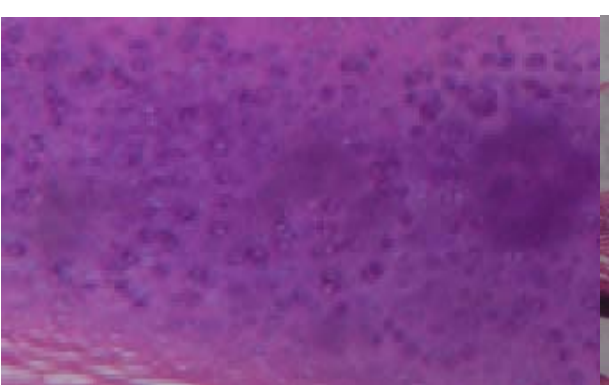

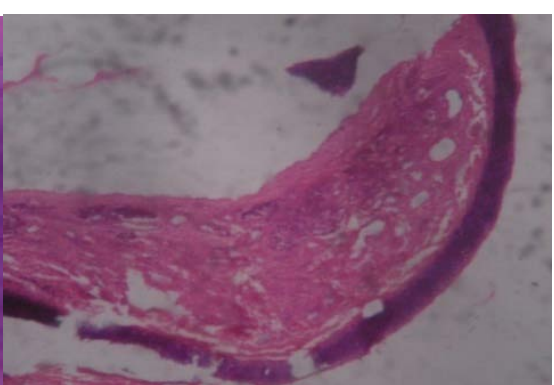

Fig. 5: [A] Nasal membrane in nanoemulsion gel [B] Nasal membrane in IPA

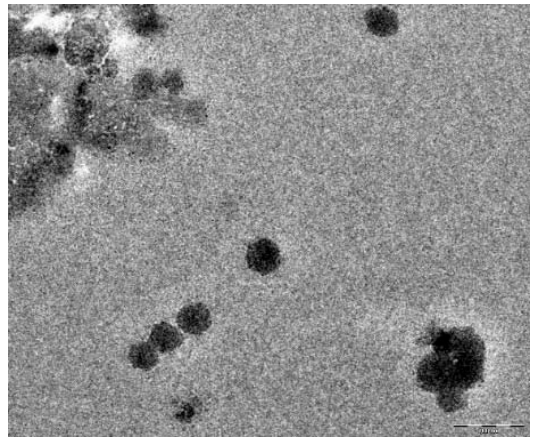

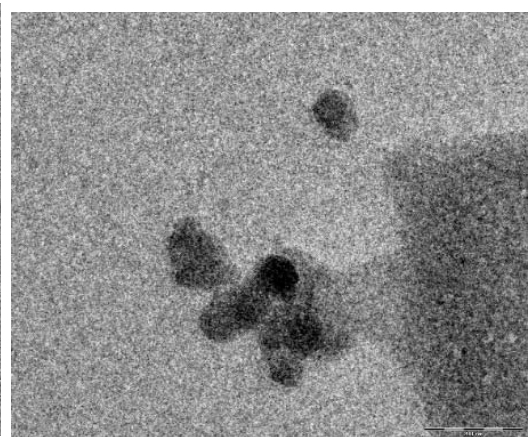

Fig. 6: Transmission electron microscopic images of (A) optimized nanoemulsion and (B) optimized in situ nanoemulsion gel 
TEM image of F5 indicated spherical shaped particles with narrow size distribution as shown in fig. 6A. F5 was formulated as in situ gel by varying the concentration of Pluronic F127 and TEM image of optimized in situ nanoemulsion gel was shown in fig. 6B.

\section{Release kinetics}

The drug release kinetic data for optimized nanoemulsion gel formulation obey the zero-order kinetic models through the regression (r2) values, which was found to be 0.998 , This may be due to the fact that the diffusion system used has a reservoir compartment (donor compartment) and goat mucosa acts as a barrier or controlling membrane; hence, the diffusion process will mimic and shall be closer to reservoir system i.e. zero-order (concentration-independent) diffusion. The corresponding plot (log \% drug release vs log time) for the Korsmeyer-Peppas equation indicated good linearity $(\mathrm{r} 2=0.999)$ for Buspirone loaded nanoemulsion gel formulation. From the Korsmeyer-Peppas equation, the release exponent value (n) for the formulation was found to be 0.954 which lies within the range, implies that the release of drug from optimized formulation obeys the nonFickian diffusion mechanism. On the basis of optimum surfactant and cosurfactant concentrations, PDI and low droplet size, the mucoadhesive nanoemulsion gel formulation of Buspirone that consist of $17.5 \% \mathrm{~W} / \mathrm{W}$ of PF 127 as thermoreversible polymer illustrated significant improvement in drug release which ensure a significant reduction in dose as well as reduces side effect by avoiding first-pass metabolism via nasal route for treatment of Anxiety disorder. The formulation was free from nasal ciliotoxicity and the approach described appears promising for brain targeting via nasal route.

\section{CONCLUSION}

The data in the present study revealed that in situ nanoemulsion gel manifest significant improvement in drug release which ensures a significant reduction in dose as well as reduces side effect by avoiding first-pass metabolism via nasal route for treatment of anxiety disorders. However, the study also has several limitations. First, appropriate instillation of formulation into the nasal cavity, another effect of the formulation containing buspirone, on neurotransmitter level require investigation in further studies. Moreover, although in vitro experiments were performed in the current study. Eventually, whether in situ nanoemulsion gel could improve bioavailability and targeting ability require further investigation.

\section{ABBRIVIATION}

GAD-Generalized anxiety disorder, BNEs-Buspirone-loaded nanoemulsions, KC cell-Keshary-Chien cell, PDI-Poly dispersity index, SLN-Solid lipid nanoparticles, NLC-Nanostructured lipid carriers, nm-Nanometer, PBS-Phosphate buffer saline, PF127Pluronic F127, IPM-Isopropyl myristate, PEG 400-Polyethylene glycol 400, NE-Nanoemulsion, Smix-Surfactant and co surfactant mixture, w/w-Weight/weight, \%-Percentage, ${ }^{\circ} \mathrm{C}$-Degree centigrade, Min-Minute, TEM-Transmission electron microscopy, ml-Mililitre, mg-Miligram, cm-Centimeter, mv-Mili volt, $\mu \mathrm{m}$-Micrometer.

\section{ACKNOWLEDGEMENT}

The authors acknowledge the support received from Dr. Rajeev Shukla and Dr. Abhishek kumar, SHEAT College of Pharmacy, Varanasi, Dr. APJ Abdul Kalam Technical University, for their support and encouragement in carrying out this research work.

\section{FUNDING}

Nil

\section{AUTHORS CONTRIBUTIONS}

All the authors have contributed equally.

\section{CONFLICT OF INTERESTS}

\section{Declared none}

\section{REFERENCES}

1. Wu C, Li B, Zhang Y, Chen T, Chen C, Jiang W, et al. Intranasal delivery of paeoniflorin nanocrystals for brain targeting. Asian J Pharm Sci 2020;15:326-35.
2. Chattopadhyay S, Das S, Sarma KN. Nose-to-brain drug delivery: an update to the alternative path to successful targeted anti-migraine drugs. Int J Appl Pharm 2021;13:67-75.

3. Kim KT, Lee JY, Park JH, Kim MH, Kim JS, Shin HJ, et al. Development of HPLC method for the determination of buspirone in rat plasma using fluorescence detection and its application to a pharmacokinetic study. Chem Pharm Bull 2016;64:1582-8.

4. Najmul Hejaz Azmi S. Determination of buspirone $\mathrm{HCl}$ in commercial dosage forms by extractive spectrophotometric method and comparison by HPLC method. Sci J Anal Chem 2015;3:91-9.

5. Shah VA, Patel JK. Optmization and characterization of doxorubicin loaded solid lipid nanosuspension for nose to brain delivery using design expert software. Int J Pharm Pharm Sci 2021;13:45-57.

6. Ingrole RSJ, Gill HS. Special section on drug delivery technologiesminireview microneedle coating methods: a review with a perspective. J Pharmacol Exp Ther 2019;370:555-69.

7. Mittal D, Ali A, Md S, Baboota S, Sahni JK, Ali J. Insights into direct nose to brain delivery: current status and future perspective. Drug Delivery 2014;21:75-86.

8. Bonferoni MC, Rossi S, Sandri G, Ferrari F, Gavini E, Rassu G, et al. Nanoemulsions for "nose-to-brain" drug delivery. Pharmaceutics 2019;1:1-17.

9. Hamid KM, Wais M, Sawant G. A review on nanoemulsion formulation, composition and application. Asian J Pharm Clin Res 2021;14;22-8.

10. Shah J, Nair AB, Jacob S, Patel RK, Shah H, Shehata TM, et al. Nanoemulsion based vehicle for effective ocular delivery of moxifloxacin using experimental design and pharmacokinetic study in rabbits. Pharmaceutics 2019;11:1-16.

11. Chuo WH, LO YK, Huang YT, WU CS. Stastistical optimization and stability study of Quercetin loaded microemulsion. Int J Pharm Pharm Sci 2021;13:23-35.

12. Ding J, Li J, Mao S. Development and evaluation of vinpocetine inclusion complex for brain targeting. Asian J Pharm Sci 2015;10:114-20.

13. Shafiq S, Shakeel F, Talegaonkar S, Ahmad FJ, Khar RK, Ali M. Development and bioavailability assessment of ramipril nanoemulsion formulation. Eur J Pharm Biopharm 2007;66:227-43.

14. Maraie NK, Almajidi YQ. Application of nanoemulsion technology for preparation and evaluation of intranasal mucoadhesive nano-in-situ gel for ondansetron HCL. J Glob Pharma Technol 2018;10:431-42.

15. Wik J, Bansal KK, Assmuth T, Rosling A, Rosenholm JM. Facile methodology of nanoemulsion preparation using oily polymer for the delivery of poorly soluble drugs. Drug Delivery Transl Res 2020;10:1228-40.

16. Harika K, Debnath S, Babu MN. Formulation and evaluation of nanoemulsion of amphotericin b. Int J Nov Trends Pharm Sci 2015;5:114-22.

17. Gaba B, Khan T, Haider MF, Alam T, Baboota S, Parvez S, et al. Vitamin $\mathrm{E}$ loaded naringenin nanoemulsion via intranasal delivery for the management of oxidative stress in a 6-OHDA Parkinson's disease model. Biomed Res Int 2019;1-20. https://doi.org/10.1155/2019/2382563

18. Jamil L, Jan SU, Gul R. Formulation of microemulsion based gel of salbutamol sulfate and its in vitro studies. Int J Curr Pharm Res 2020;12:102-7.

19. Pengon S, Chinatangkul N, Limmatvapirat C, Limmatvapirat $S$. The effect of surfactant on the physical properties of coconut oil nanoemulsions. Asian J Pharm Sci 2018;13:409-14.

20. Mustafa IF, Hussein MZ. Synthesis and technology of nanoemulsion-based pesticide formulation. Nanomaterials 2020;10:1-26.

21. Sharma D, Maheshwari D, Philip G, Rana R, Bhatia S, Singh M, et al. Formulation and optimization of polymeric nanoparticles for intranasal delivery of lorazepam using box-behnken design: in vitro and in vivo evaluation. Biomed Res Int 2014;1-14. DOI:10.1155/2014/156010

22. Garala K, Joshi P, Patel J, Ramkishan A, Shah M. Formulation and evaluation of periodontal in situ gel. Int J Pharm Investig 2013;3:29-41. 
23. Aderibigbe BA, Naki T. Chitosan-based nanocarriers for nose to brain delivery. Appl Sci 2019;9:1-27.

24. Kaur P, Garg T, Vaidya B, Prakash A, Rath G, Goyal AK. Brain delivery of intranasal in situ gel of nanoparticulated polymeric carriers containing antidepressant drug: behavioral and biochemical assessment. J Drug Target 2015;23:275-86.

25. Jaiswal M, Kumar M, Pathak K. Zero order delivery of itraconazole via polymeric micelles incorporated in situ ocular gel for the management of fungal keratitis. Colloids Surfaces B 2015;130:23-30.

26. Zeng L, Liu Y, Pan J, Liu X. Formulation and evaluation of norcanthridin nanoemulsions against the plutella xylostella (Lepidotera: Plutellidae). BMC Biotechnol 2019;19:1-11.

27. Patel RB, Patel MR, Bhatt KK, Patel BG, Gaikwad RV. Evaluation of brain targeting efficiency of intranasal microemulsion containing olanzapine: pharmacodynamic and pharmacokinetic consideration. Drug Delivery 2016;23:30715.

28. Sudheer P. Design and evaluation of a self-emulsifying drug delivery system of aripiprazole. Int J Pharm Sci 2019;81:108998.

29. Xu M, Mou Y, Hu M, Dong W, Su X, Wu R, et al. Evaluation of micelles incorporated into thermosensitive hydrogels for intratumoral delivery and controlled release of docetaxel: a dual approach for in situ treatment of tumors. Asian J Pharm Sci 2018;13:373-82.

30. Sachan AK, Tripathi K, Sachan P, Gangwar SS. Fabrication and characterization of compressed solid dispersion based fast dissolving tablets of sildenafil citrate. Int J Pharm Sci Rev Res 2015;30:98-104. 